\title{
Cardiac Motion Extraction in Multislice Computed Tomography by Using a 3D Hierarchical Surface Matching Process
}

\author{
M Garreau ${ }^{1}$, A Simon ${ }^{1}$, D Boulmier ${ }^{2}$, H Guillaume $^{1}$ \\ ${ }^{1}$ LTSI, INSERM U642, Université de Rennes 1, Campus de Beaulieu, 35042 Rennes, France \\ ${ }^{2}$ Centre Cardio-Pneumologique, CHU Pontchaillou, 35033 Rennes, France
}

\begin{abstract}
Retrospective data reconstruction provided in Multislice Computed Tomography (MSCT) scanners offers new perspectives for cardiac kinetics evaluation with volume sequences of high spatial and temporal resolutions. A new method is proposed for cardiac motion extraction in MSCT. It is based on a 3D surface matching process associated to a hierarchical description of shapes. It provides $3 D$ velocity fields associated to the left ventricle inner surface, which can be used for global and local motion quantification. $3 D$ segmentation and surface reconstruction processes are first applied on each volume to obtain one mesh representation for each time. A hierarchical surface matching scheme is then performed: mesh nodes are selected as entities and matched according to a local energy. A simulated annealing is used to perform a global optimisation of the correspondences. First results obtained on simulated and real data show the good behavior of this method.
\end{abstract}

\section{Introduction}

Cardiovascular diseases are a major cause of mortality, being responsible for about 30\% of registered adult deaths in industrial countries. Because a more sensitive measurement of myocardial function might result in earlier diagnosis, more effective treatment of heart disease may be possible. Technological improvements in cardiac imaging provide rich opportunities for such progress. It is in particular the case with spiral computed tomography, with the introduction of ultra-fast rotating gantries (-0.5s/tr-) along with multi-rows detectors, higher spatial and temporal resolutions, retrospective ECG-gated reconstructions. Some reference studies have been conducted in MSCT for the detection of coronary diseases $[1,2]$, but not yet on the quantitative $3 \mathrm{D}$ cardiac motion extraction.

The issue of nonrigid motion estimation from 3D images is one of the most important challenges of computer vision.
Methods which have been proposed for this purpose are classified into three kinds of approaches: geometric deformable models, optical flow computation and feature matching methods.

In geometric model-based approaches, parametric models $[3,4]$ involve the parametric formulation of the object and/or of the movement. This kind of method is interesting to extract global motion and to represent it with few parameters. Non parametric models [5, 6], using mainly mass-spring and finite element methods, extract local motion using differential constraints. The main difficulty of these approaches is to capture precision enough to take advantage of the fine description of objects provided by MSCT. Optical flow methods [7, 8] are mainly based on intensity conservation and motion smoothing constraints. That constraint of intensity conservation with time is difficult to advance with MSCT data because of the contrast agent diffusion combined with the retrospective reconstruction of the sequence. Furthermore, these methods providing dense motion fields are not well adapted to data volumes of important size in which the study deals only with few objects. Feature matching methods $[9,10,11]$ are based on the search of correpondences between entities (considered at $t$ and $t+1$ times) according to descriptive parameters. These methods enable to concentrate the study on the objects of interest and to extract local and global motion.

We propose a method to extract elastic motion from cardiac MSCT images. This approach is based on local 3D hierarchical surface feature matching, under global constraints using simulated annealing algorithm. First results obtained on simulated and real data show the great potential of this new modality for cardiac motion evaluation in non invasive imaging.

In section 2, we first describe the developped feature matching method, applied at one resolution. Then we explain how this process is adapted using a multiresolution scheme. In section 3 we present the results obtained on simulated and real data. In section 4 we conclude and discuss about the issues to develop in further 
research.

\section{Method}

The motion extraction method we propose needs in a first step a 3D segmentation stage. This detection part is based on a region growing process constrained by contour information and provides the segmented surface of the object [12].

The next issue is to compute the displacement vector field associated to $3 \mathrm{D}$ objects between two successive frames. The structures of interest in these images are nonrigid and verify some consistency in shape and motion. This motion estimation is achieved by a local search of correspondences between 3D surface meshes which represent the extracted objects at each time of the sequence.

The feature matching methods rely on the matching of specific entities according to some descriptive parameters. A local energy based on these parameters is defined to compare two entities considered at successive times $t$ and $t+1$. A minimization process is then applied to globally optimize the correspondences.

Using that kind of methods involves three steps:

- the choice of the entities nature and of their descriptive parameters,

- the definition of the local energy,

- the choice of the optimization process.

In next paragraphs we describe how we have dealt with each of these issues and we highlight how the process has been developed using the benefits of a hierarchical scheme.

\subsection{Choice of the entities and of their descriptive parameters}

Two main classes of entities can be identified: entities which are focused on particular features of the object and entities which describe the whole object of interest.

We have focused our study on 3D mesh nodes, allowing to precisely describe a surface mesh, to compute a hierarchical description of the surface, and to give the possibility to use different surface descriptors.

The topological configuration of local surfaces can be described by different parameters such as the surface orientation, the mean or gaussian curvature. In this work the mean curvature has been selected and provided by the integral absolute mean curvature [13] which gives information on the flatness of the nodes neighborhood.

\subsection{Definition of the local energy}

A local energy function is defined to match two mesh nodes (one at time $t$, the other at time $t+1$ ). This function is obtained as the linear combination of two terms (eq. 1) :

$$
E_{\text {Locale }}(a, b)=\beta_{1} \cdot E_{\text {Data }}(a, b)+\beta_{2} \cdot E_{\text {reg }}(a, b)
$$

where $a$ and $b$ represent the two nodes; $E_{D a t a}(a, b)$ is a data energy term which represents a distance between the estimated motion and the data observed; $E_{\text {reg }}(a, b)$ is a spatio-temporal regularity constraint; $\beta_{1}$ and $\beta_{2}$ are two weighting coefficients in order to balance the importance of each term.

The data fidelity term is defined according to the nodes coordinates and to their descriptive parameters. It is given by :

$$
E_{\text {Data }}(a, b)=\alpha_{\text {Dist }} . U_{D i s t}(a, b)+\alpha_{\text {Topo }} . U_{\text {Topo }}(a, b)
$$

where $U_{\text {Dist }}(a, b)$ provides the euclidian distance between two nodes; $U_{T o p o}(a, b)$ is related to the topological information based on mean curvature; $\alpha_{D i s t}$ and $\alpha_{\text {Topo }}$ are weighting factors.

The regularization term prevents to obtain aberrant results and enables to compute smooth correspondence field to preserve spatial and temporal coherence. This second energy term is based on neighborhood constraints about correspondences of the current node and of its neighborhing nodes.

\subsection{Optimization}

The computation of the displacement vector field is obtained by the minimization of a global energy, providing correspondences of highest probability. The simulated annealing algorithm has been used in order to minimize the global energy resulted from the summation of local energies. From an initial configuration of the motion field, one iteration consists in the selection of a set of nodes in a random way, the analysis of possibilities to accept a transition (modification of correspondence) for each selected node and the progressive linear lowering of a temperature parameter. The computation cost of this process is limited by the fact that the transition of one node affects only the local energy associated to this node. The process is stopped when the temperature reachs zero.

\subsection{Multi-resolution process}

Taking advantage of the spatial resolution of the volumes acquired with MSCT, a hierarchical scheme is used to optimize the convergence of the matching algoritm, to reduce the computation time and to take advantage of the spatial resolution provided by MSCT. In fact, the complexity of the segmented surface highlights the need to describe topological features at different resolutions in order to match entities according to different levels of details. 
To obtain the surface at one resolution, the segmented volume at the previous resolution is first filtered (with a gaussian filter) and is down-sampled by a factor 2 . Finally, a surface reconstruction process is applied to the resulting volume to obtain the surface at desired resolution.

The matching process is first applied at the lowest resolution to take the coarsest details into account (the initialization of correspondences is then done by the selection of the nearest node at $t+1$ ). For the computation of correspondences at next resolution, the result of that optimization is used as an initialization, after an interpolation process: for each node, the neighboring nodes at lower resolution are selected; the average of their estimated motion is computed; the chosen node at next resolution is selected as the node corresponding to a movement with the nearest direction and amplitude.

This process is applied iteratively until the correspondences at desired resolution are obtained.

\section{Results}

\subsection{Tests on simulated data}

One simulated model has been used to test the matching process between two successive moments. The model is composed of a superellipsoidal shape deformed by three kinds of movement: local deformation, twisting deformation and expansion/compression deformation. To obtain the model corresponding to the image at $t$ time, the original shape is iteratively locally deformed to render the complexity of the endocardium (cf Fig. 1). This surface is next deformed with the three kinds of motion to compute the model corresponding to the image at $t+1$ time.

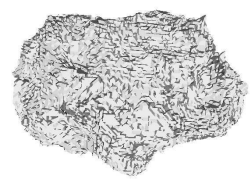

Figure 1. Simulated model.

The motion extraction process has been tested with this model, using three hierarchical resolutions and the three kinds of motion applied simultaneously. The process enables to obtain a mean error of the estimated motion of 0.5 voxel (with an error inferior to 1 voxel for $85 \%$ of nodes).

\subsection{Results on real data}

The algorithm has been also tested on real human heart data with a temporal database acquired by a Siemens SOMATOM Sensation 16 with ten volume images representing a whole cardiac cycle. The segmentation preprocess has been applied to each volume of the sequence resulting to the segmentation of the left cavities of the heart and of the beginning of the aorta. To obtain surface meshes, segmented volumes are then processed by a surface recontruction tool, such as the Marching Cubes algorithm.

The descriptor used to match two nodes, the integral absolute mean curvature, has highlighted a spatial and temporal coherence and so can be used to compare along time the local topology of two nodes.

The multi-resolution motion extraction process has been applied to the sequence, each time between two successive endocardial surfaces. The minimal resolution used is obtained from the volume image (of dimension $512 \times 512 \times 300$ ) after four consecutive filtering and downsampling steps. Three levels of resolution of the images are presented on Fig. 2. The global correspondence matching
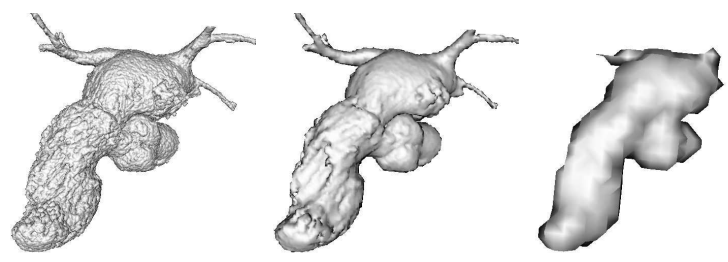

Figure 2. Surface at three resolutions (respectively the higher resolution and after two and four filtering and downsampling processes).

process results, at each resolution, in a set of displacement vectors defined on each node of the mesh. The parameters concerning the initial temperature and weighting factors of the energy function have been chosen by an empirical way after successive tests.

As illustrations, Fig. 3 shows the estimated motion amplitude at two resolutions for one instant corresponding to the beginning of the systole. The contraction movements

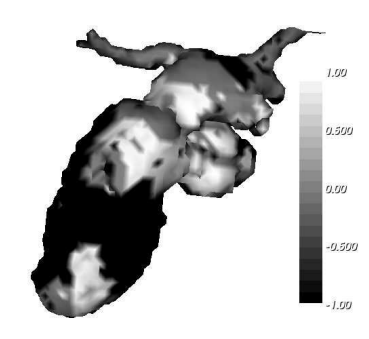

(a)

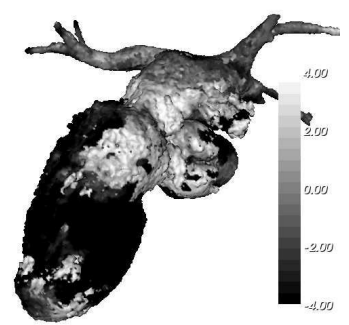

(b)
Figure 3. Estimated motion amplitude at the beginning of the systole at two resolutions ((a) after three and (b) after one filtering and downsampling processes) (colors: in white: expansion, black: contraction).

(represented with black color), characteristic of systole, are well identified. At lowest resolution, surface and movements are coarse but highlight the main motion. 
Movements represented at higher resolution, coherent with results at previous resolution, are more precise and enable to deal with local motion.

Fig. 4 shows the estimated motion amplitude at the higher resolution, at two moments of the cardiac cycle: beginning of the diastole and beginning of the systole.

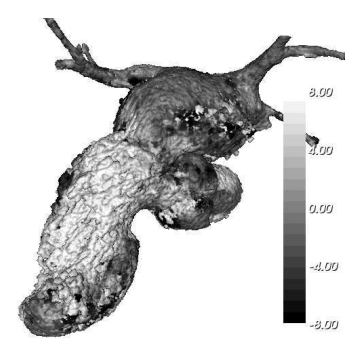

(a)

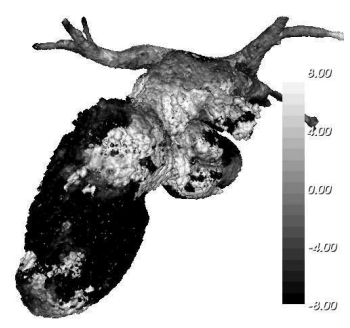

(b)
Figure 4. Estimated motion amplitude at (a) the beginning of the diastole and (b) the beginning of the systole (colors: in white: expansion, black: contraction).

These results show expansion during the diastole (in white colour) and contraction during the systole and are coherent with the cardiac phases. From this set of 3D vectors, some local descriptive parameters related to cardiac dynamics can be computed such as motion amplitude and direction of anatomical segments or motion direction according to time. These parameters will allow to extract clinical informations. As an example, Fig. 4 highlights the pathological situation where the apical area suffers from akinesia.

\section{Conclusions}

A first solution has been proposed for the 3D motion extraction of the left ventricle in cardiac MSCT images. Our approach is applied on extracted volumes, using a hierarchical 3D feature matching method and provides first 3D velocity fields for the left cavity surface. These displacement vectors can represent accurate informations related to contraction and expansion movements of anatomical segments. These first results confirm the great potential of MSCT imaging for cardiac applications.

\section{Acknowledgements}

This work is supported by Brittany region and the French Medical Research and Health National Institute (INSERM). The authors express their thanks to Siemens, Medical Division, France.

\section{References}

[1] Schroeder S, Kopp A, Ohnesorge B, Flohr T, Baumbach A, Kuettner A, Herdeg C, Karsch K, Claussen C. Accuracy and reliability of quantitative measurements in coronary arteries by multi-slice computed tomography: Experimental and initial clinical results. Clinical Radiology 2001;56:466474.

[2] Larralde A, Boldak C, Garreau M, Toumoulin C, Boulmier D, Rolland Y. Evaluation of a 3D segmentation for coronary artery characterization in multi-slice computed tomography. In 2nd International Conf. on Functional Imaging and Modeling of the Heart, FIMH'2003. 2003; 39-51.

[3] Bardinet E, Cohen LD, Ayache N. Tracking and motion analysis of the left ventricle with deformable superquadrics. Medical Image Analysis 1996;1(2):129-49.

[4] Chen C, Huang T, Arrott M. Modeling, analysis and visualization of left ventricle shape and motion by hierarchical decomposition. IEEE Transactions on Pattern Analysis and Machine Intelligence 1994;16(4):342-356.

[5] Choi SM, Kim MH. Motion visualization of human left ventricle with a time-varying deformable model for cardiac diagnosis. The journal of visualization and computer animation 2001;12:55-66.

[6] Benayoun S, Nastar C, Ayache N. Dense nonrigid motion estimation in sequences of 3D images using differential constraints. Lecture Notes in Computer Science Computer Vision Virtual Reality and Robotics in Medicine Apr. 1995 1995;309-318.

[7] Song SM, Leahy RM. Computation of 3-D velocity fields from 3-D cine CT images of the human heart. IEEE Transactions on Medical Imaging 1991;10(3):295-306.

[8] Gorce JM, Friboulet D, Magnin IE. Estimation of three-dimensional cardiac velocity fields: assessment of a differential method and application to three-dimensional CT data. Medical Image Analysis 1997;1(3):245-61.

[9] Amini AA, Duncan JS. Bending and stretching models for LV wall motion analysis from curves and surfaces. Image and Vision Computing 1992;10(6):418-430.

[10] Kambhamettu C, Goldgof D, He M, Laskov P. 3D nonrigid motion analysis under small deformations. Image and Vision Computing 2003;21(3):229-245.

[11] Shi P, Sinusas A, Constable R, Ritman E, Duncan JS. Pointtracked quantitative analysis of left ventricular motion from 3D image sequences. IEEE Transactions on Medical Imaging 2000;19(1):36-50.

[12] Guillaume H, Garreau M. Segmentation de cavités cardiaques en imagerie scanner multi-barettes. In 12ème Forum des Jeunes Chercheurs en Génie Biologique et Médical, Nantes, France; 2003.

[13] Dyn N, Hormann K, Kim SJ, Levin D. Optimizing 3D triangulations using discrete curvature analysis. In Mathematical Methods for Curves and Surfaces. Nashville, TN: Vanderbilt University Press; 2000.

Address for correspondence:

Mireille Garreau

LTSI, INSERM U642, Université de Rennes 1,

Campus de Beaulieu, 35042 Rennes, France

mireille.garreau@univ-rennes1.fr 